\title{
A Method of Noise Control using in Shearography Inspection System
}

\author{
ZHANG Jian ${ }^{1,2, a}$, FENG Huijuan ${ }^{2, b}$ and WANG Xiaoyan ${ }^{2, c}$ \\ ${ }^{1}$ University of Science and Technology of China, Hefei 230027, CHINA \\ ${ }^{2}$ Military Transportation University, Tianjin 300161, CHINA \\ ayitzhak@163.com, bbluebellfhj@163.com, wangxy_y@163.com
}

Keywords: Noise Control, Inspection, Wavelet

\begin{abstract}
The shearography inspection technology is extremely fitting for the non-destructive testing of composites, tyres, panels of vehicle or airplane. But the testing image of shearography includes much speckle noise, so noise control is a very important task. A method called real time integrated porcessing is presented is this paper, especially, wavelet transform method is multi-resolution analysis, it can reduce noise, and at the same time, can keep the details of the image. The wavelet noise control technique is studied in this paper particularly. Experimental results are satisfactory.
\end{abstract}

\section{Introduction}

Shearography is a laser-based interferometry in conjunction with the digital imaging processing technique for full-field measurement of surface deformation, such as composites, tyres, panels of vehicle or airplane. It reveals defects in an object by looking for defect-induced deformation anomalies. It does not require special vibration isolation, and with the development of a small and mobile measuring device(portable inspection system), it can be employed easily in field/factory environments ${ }^{[1]}$.

The potential advantages of shearography inspection are: (a) rapid large area inspection; (b) non-contact testing without immersion of the component or the use of water jets; (c) ability to inspect regions that attenuate or scatter ultrasound; (d) ability to indicate structural strength in addition to providing passive defect detection ${ }^{[2]}$.

The readability of detecting image impacts the decision of defects in material. There is much noises in inspecting images, so, the noise control of detecting image is a very important question.

The traditional noise control method uses the low-pass filtering. But the resolution cannot change, so the detail of the image is lost. The wavelet transform can keep the image details, at the same time, it can reduce the noise. A method called real time integrated porcessing is presented is this paper, this method includes a core technique, which is wavelet transform. The wavelet noise control technique is particularly studied in this paper.

A new highly mobile inspection system was built, and some test was conducted by using this epuipment. A Software was developed by using the method called real time integrated porcessing, we compare the result of original inspection result and processed inspection result, the later's result can be recognized easily.

\section{Shearography inspection system}

Speckle shearography is a reliable coherent-optical method for the nondestructive testing of technical components with respect to surface and sub-surface flaws. In shearography systems, the object under investigation is illuminated by an expanded laser beam, forming a speckle pattern if the object surface is optically rough. The speckle pattern is optically processed by viewing through a shearing interferometer, and the resulting interferogram is recorded by an electronic sensor (e.g. CCD camera). Speckle interferograms, recorded before and after object deformation, are correlated, using a PC, to yield correlation fringes. The phase of these fringes is sensitive to the displacement gradient. 
Real time shearography is performed after the operator captures the first image. Each successive image is compared with this first image. As the object is stressed during the test, the strain changes in the object from the first are displayed as fringes defining the size, shape, and location of the defect or the amount of plane strain. When the operator decides to "freeze" the real time test results, comparing the first image of the test object with these frozen $\mathrm{N}$ image, is displayed. Debonded areas in a honeycomb panel will expand out of the plane of the panel, letting the shearographic interferometer detect this localized deformation. The test sequence may be programmed allowing exact replication of the application of the stress level during test ${ }^{[3]}$.

In the cases of vehicle or aircraft inspection and maintenance, a portable shearography inspection system is very useful. We has successfully developed an inspection system. In figure 1 and figure 2, such a system is presented.

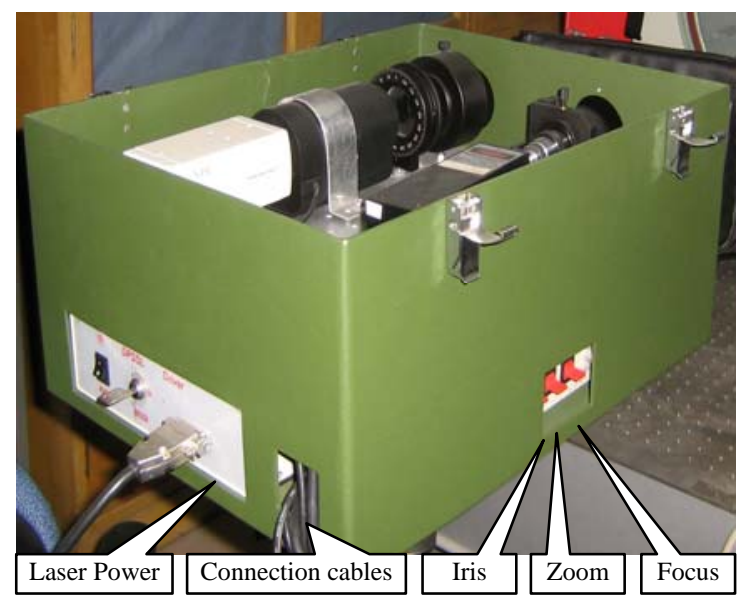

Fig. 1. Outlook of the portable inspection system

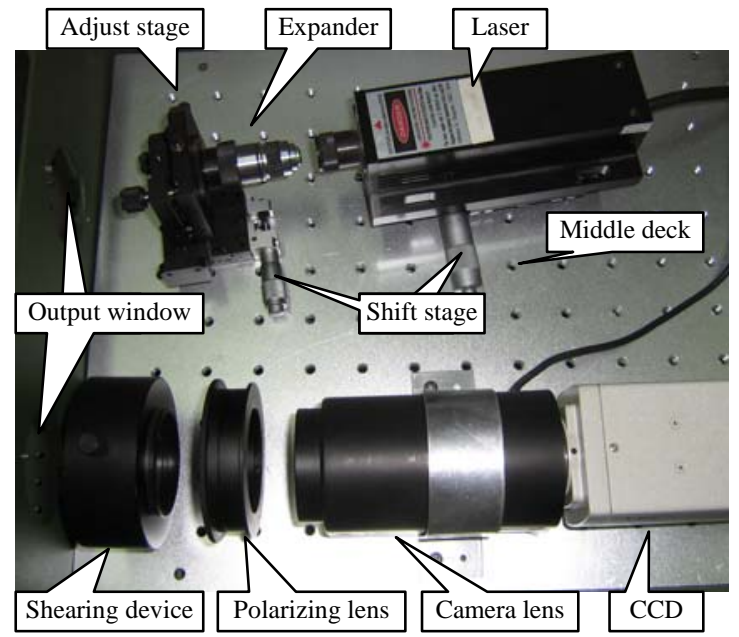

Fig. 2. Layout of upper layer of the portable inspection system

It consists of a heating lamp, which shines directly to the surface to be inspected. Debondings or other defects will show up in typical deformation patterns. The operator can view the inspection result with a notebook pc and therefore is flexible to operate on scaffolds or inside openings.

\section{Noise Control Method}

The shearography testing result image contains much noises, inspector cannot recognize the defects easilly, so we must control the noises of the image. A new method called real time integrated noise control was presented is this paper. Many image process methods is used in sequence. Figure 3 shows the flow of the process.

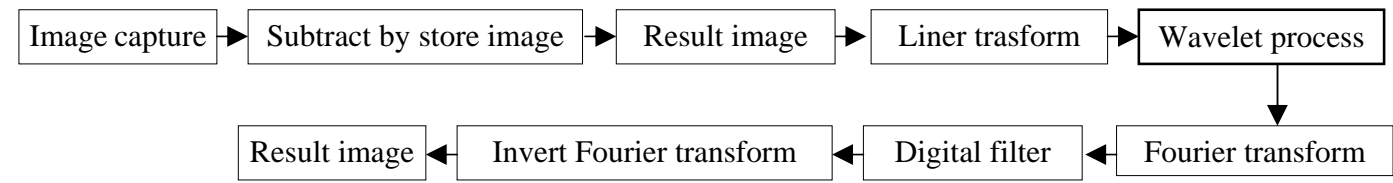

Fig. 3. Flow chart of integrated denoising method

Among the techniques shown in figure 3, the wavelet process is the core technique. The thought of wavelet analysis is from the method of multi-resolution. French scientist Molet put forward the concept of wavelet in 1984. Through the work of Meyer, Grossmann, Daubechies and Mallat, the wavelet analysis method is built.

Mother wavelet function $\psi(x)$ can be changed into a wavelet sequence $\psi_{a, b}(x)$ : 


$$
\psi_{a, b}(x)=|a|^{-\frac{1}{2}} \psi\left(\frac{x-b}{a}\right), \quad a, b \in R, a \neq 0
$$

The wavelet transform of function $f(x)$ is:

$$
\begin{aligned}
W_{f}(a, b) & =\int_{-\infty}^{+\infty} f(x) \psi_{a, b}^{*}(x) d x \\
& =\left\langle f(x), \psi_{a, b}(x)\right\rangle
\end{aligned}
$$

$a$ - dilation factor

$b$ - translation factor

The essence of wavelet transformation is expanding the signal on the mother wavelet. The dissolution diagram is shown in figure 4. Figure 5 shows the principle of wavelet transform denoising.

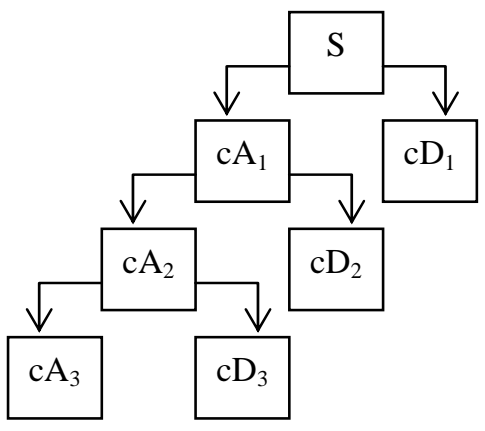

Fig. 4 Dissolution diagram of wavelet

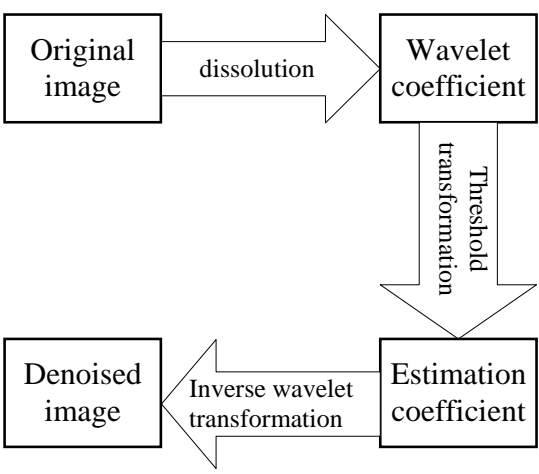

Fig. 5 Chart of principle of wavelet denoising

In figure $4, \mathrm{~S}$ is the signal to be resolved, $c A_{i}$ is the approximation coefficient. It reflects the low frequency part. $c D_{i}$ is the detail coefficient, it reflects the high frequency part.

The characteristics of wavelet transformation are de-correlation, multi-resolution ${ }^{[4]}$. The basic principle of wavelet transformation denoising is choosing an appropriate threshold or threshold function to transform the wavelet coefficient, then inverse transforming the handled coefficient to rebuild image $\mathrm{e}^{[5]}$.

In the field of wavelet transformation, the bigger coefficients are useful information, the smaller coefficients are noise information.

\section{Testing}

Wollaston prism inspection system is designed in this paper, the type of defect is debonding, the specification of test specimen is : $350 \mathrm{~mm} \times 180 \mathrm{~mm} \times 25 \mathrm{~mm}$. The defect size is $10 \mathrm{~mm}$. The loading method is vacuum. The original inspection result image is $768 \times 576$ pixels, shown in figure 6 .

The result image after real time integrated processing is shown in figure 7.

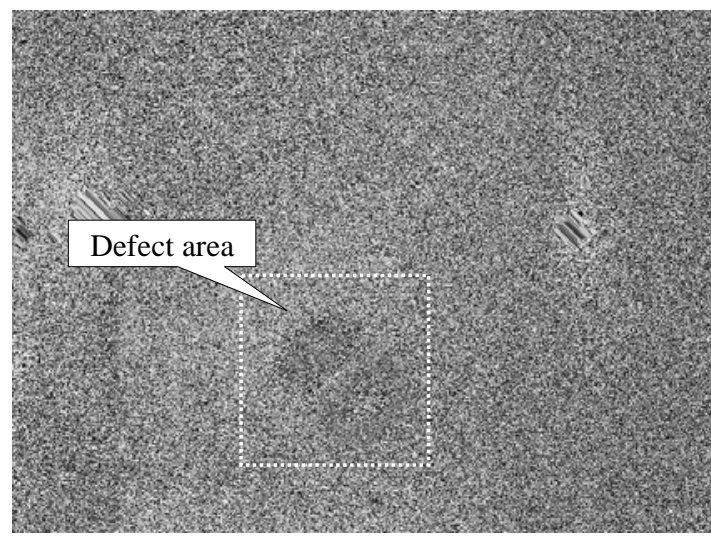

Fig. 6 Original inspection result image

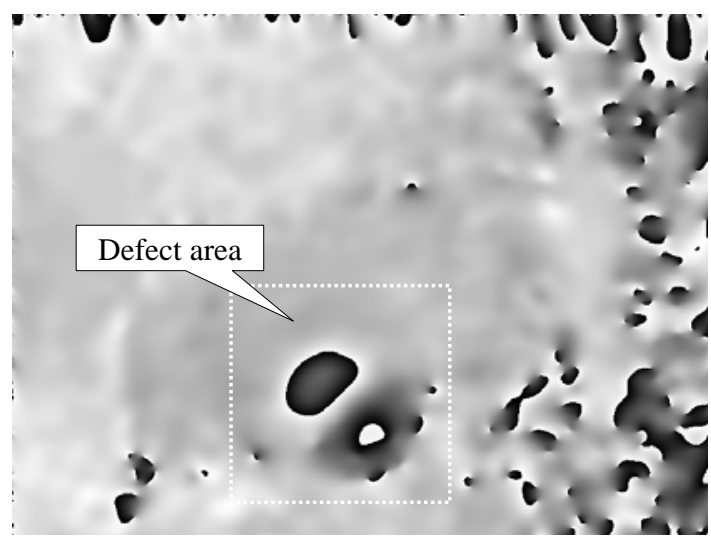

Fig. 7 Result image after processing 
Through the comparing of several wavelets, the Daubechies 4(db4) wavelet is chosen in this paper. If the dissolution level is higher, the computing time cannot be accepted. If the dissolution is lower, the effects is not good, so the dissolution level we chose is 4 level.

In figure 6, due to the noise, the readability of defect is not acceptable. After using the noise control method called real time integrated processing, the readability of the result image shown in figure 7 is very satisfactory. The inspector who is not be trained can recognize the defect easily.

\section{Conclusion}

A new highly mobile inspection method has been introduced into the world of in service inspection for maintenance of tyres, panels of vehicle or aircrafts and components under rough industrial conditions. Shearography can deal with harsh environmental conditions, can be used really under daylight conditions and still can give precise indications of flaws in a wide variety of inspection situations.

A method called real time integrated processing is presented is this paper. This method includes several technique of noise control, wavelet transform technique is particularly studied in this paper. Through the testing result, this method is proved to be very efficient.

\section{References}

[1] K. Habib. Critical assessment of the integrity of thin organic films by shearography. Journal of Failure Analysis and Prevention. Vol. 10(2010), p427.

[2] M. Kalms, W. Osten. Advanced shearographic system for non-destructive testing of industrial and artwork components. Proceedings of SPIE Vol. 4915(2002), p34.

[3] Y. Y. Hung. Digital Shearography versus TV-Holography for Non-Destructive Evaluation [C]. Optics and Lasers in Engineering. Vol. 26(1997), p422.

[4] B. Vidakovic, C. B. Lozoya. On time-dependent wavelet denoising[J]. IEEE Trans. Signal Processing, Vol. 46(1998), p254.

[5] I. S. Bozchalooi , M. Liang. A smoothness index guided approach to wavelet parameter selection in signal de-noising and fault detection [J]. Journal of Sound and Vibration, Vol. 122(2007), p247. 